\title{
Egocentric organization of spatial activities in imagined navigation
}

\author{
MARIOS N. AVRAAMIDES and RICHARD A. CARLSON \\ Pennsylvania State University, University Park, Pennsylvania
}

\begin{abstract}
Studies on spatial frameworks suggest that the way we locate objects in imagined environments is influenced by the physical and functional properties of the world and our body. The present study provides evidence that such an influence also characterizesimagined navigation. In Experiment 1, participants followed spatial directions to construct an imagined path, while either keeping constant or updating their orientation at each step. A pattern of step times diagnostic of spatial frameworks was obtained in the updated-orientation but not in the constant-orientation condition. In Experiment 2, participants performed the updated-orientation condition with two levels of external support for the reference frame being used. Step times conformed to the predictions of spatial frameworks in both conditions. Both experiments also provided support that the processes involved in imagined navigation exhibit the operator-operand dynamics of other mental skills previously documented in the mental arithmetic domain. These results reinforce Piaget's (1954) notion that spatial displacements and integer arithmetic share a set of structural characteristics.
\end{abstract}

Tasks involving imagined or depicted situations often evoke mental operations that are similar to-and presumably derived from, either in development or in evolutionthe perceptual-motor operations that apply to real situations (Jeannerod, 1995; Kosslyn, 1994). For example, mental operations on visual images appear to share properties with perception of the environment (e.g., Kosslyn, 1987, 1994). The evidence for such shared properties comes from studies demonstrating qualitatively similar effects of independent variables on both kinds of processes, as in the effect of distance on scanning in perception and in visual imagery. Sometimes, though, important differences indicate specific properties of mental operations and mental representations. For example, Tversky and her colleagues (e.g., Bryant, Tversky, \& Franklin, 1992; Franklin $\&$ Tversky, 1990) demonstrated in a series of studies that the time that participants required in order to locate objects in imagined or remembered spaces depends not on the distance one would have to turn to perceive those objects, but on the body-centered axes on which the objects are located. Thus, locating an object behind one's imagined location (using the front-back axis) is faster than locating an object to the left or right of the imagined location, although turning to view it would take longer.

In everyday life, the use of spatial terms such as front and back is not limited to locating objects in the environ-

We thank Cathleen Moore, Frank Ritter, Nancy Franklin, Ruth Maki, and David Bryant for valuable comments on previous drafts of the article. We also thank Brian Place and Jessie Carboni for valuable help with data collection. Correspondence should be sent to M. N. Avraamides, Department of Psychology, University of California, Santa Barbara, CA 93106-9660(e-mail: avraamides@ psych.ucsb.edu). ment. These terms are also frequently used for guiding locomotion. If spatial language reflects the internalized regularities of our physical and perceptual world, one would expect that the asymmetries reported when spatial terms are used for locating objects would generalize to other spatial actions, such as following spatial instructions to construct a path.

In the present experiments, we examined the properties of mental operations needed to perform a laboratory version of a common spatial task: constructing a path by following spatial instructions. Participants in these experiments imagined a path through a grid displayed on a computer screen, mentally following instructions such as "move left three squares." To perform this task successfully, they had to remember intermediate locations while interpreting the instructions at each step. We asked two questions about the mental operations required for this task. First, would the pattern of step-by-step latencies reflect the pattern found in studies of imagined spaces reported by Franklin and Tversky (1990) and Bryant et al. (1992)? Second, would the structure of mental operations in individual steps correspond to that observed in earlier studies for symbolic and spatial tasks (Carlson \& Sohn, 2000; Sohn \& Carlson, 1998)?

\section{Spatial Frameworks for Imagined Spaces}

Our study extends previous research on imagined spaces by examining performance of spatial actions rather than spatial judgments. Like real-life navigation, sequences of these actions have a cascaded character, in that the result of one action determines the starting point (location and orientation) of the next. The spatial frames of reference used to perform these actions must be centered on the participants' imagined locations and, in some of our experi- 
mental conditions, must be organized on the basis of the participants' imagined orientations.

Tversky and her colleagues (e.g., Bryant, Lanca, \& Tversky, 1995; Bryant et al., 1992; Bryant \& Wright, 1999; Franklin \& Tversky, 1990) have examined how people use the space around the body. In their typical paradigm, participants read narratives that describe themselves as central characters in settings where objects are located at the extensions of their body axes (head/feet, front/back, left/right). Probed with a direction, participants are then asked to indicate the name of the object that occupies that direction (or vice versa). Typical results, in the case of an upright observer, show that people are fastest reporting objects located on the head/feet axis, intermediately fast with objects on the front/back axis, and slowest reporting objects on the left/right axis. There is also an asymmetry within the front/back axis, such that objects in front of the central character are associated with faster responses than are objects at the back.

Franklin and Tversky (1990; see also Bryant \& Wright, 1999) proposed the spatial framework model to account for the observed pattern of data. According to this model, participants use the extensions of the three basic body axes to construct a mental spatial framework, to which they associate the objects. The perceptual and functional properties of the body and the asymmetries of the world determine the relative salience of the three axes in terms of how useful they are for localizing objects in the environment (Clark, 1973). Objects located on the three axes are differentially accessible depending on the salience level of each axis. The head/feet axis is highly asymmetric because of gravity. Moreover, distinct body parts are associated with the two poles of this axis. This asymmetry causes the head/feet axis to become the most accessible axis for localizing objects in the environment.

The front/back axis is also asymmetric since it distinguishes the part of the world we can easily sense and interact with from the rest of the world. Our perceptual organs are oriented toward the front direction, the constraints of our joints enable easier movements of our hands toward the front, and we typically move in the forward direction and interact with other persons and objects that are in front of us. This asymmetry biases our spatial reasoning so that the front direction is more accessible than the back. Despite the asymmetry provided from the physical structure of our bodies, the front/back axis lacks any support from the environment, and its orientation changes frequently as we move in the environment. This makes the front/back axis somewhat harder to access than the head/feet axis. Finally, because the left/right axis is highly symmetric (Corballis \& Beale, 1976), it becomes the least useful axis for object localization and therefore the least accessible. In fact, the difficulty people have with the left/right axis has been documented in a number of studies (e.g., Maki, 1979; Maki, Grandy, \& Hauge, 1979).

The spatial framework model provides a reasonable account for how people keep track of objects that occupy the space around the body. It is also reasonable to suppose that the differential accessibility of axes proposed in this model would affect the use of spatial directions to guide action, as in the path construction task used here. However, Tversky, Morrison, Franklin, and Bryant (1999) distinguish between the space that is described by spatial frameworks and a space for navigation. The space of navigation refers to environments that are too large to view from a single standpoint, and it is conceptualized as a two-dimensional maplike representation that incorporates information gathered from various standpoints and sources. In our study, imagined navigation takes place in a small and perceptually simple space that contains no discriminating landmarks. We therefore hypothesized that the pattern of latencies observed in the present experiments would be best accounted for by the spatial framework model.

\section{Structure of Intentions}

Navigation, real or imagined, differs from spatial judgment in that it involves a series of actions that may change the relation of one's egocentric frame of reference to external frames. When spatial instructions are given with reference to one's egocentric frame-for example, "Go left"- actions based on these instructions will have different relations to an external frame of reference depending on one's current heading. The action has the consequences both of movement and of updating one's orientation relative to the external frame. The updated orientation then provides a new egocentric frame for interpreting subsequent directions. In contrast, spatial instructions given with reference to an external frame (e.g., "Go north") do not depend on updated orientations for their interpretation.

Extending the spatial frameworks account from spatial judgment to imagined navigation suggests that spatial actions as well as spatial representations are organized in terms of axes. As Piaget (1954; also see Chapman, 1988) proposed, the organization of spatial actions constitutes a system that shares structural properties with mental operations in symbolic domains such as arithmetic. Arithmetic, of course, can be represented as movement in a unidimensional space (e.g., a number line). The set of directions, like the set of arithmetic operations, constitutes a system of symmetrical and reversible actions, a criterion in Piagetian theory for operational status (Chapman, 1988). In contrast, the set of numbers (here, integers) taken by itself does not constitute an operational system in this sense, although it serves as a medium over which operations can be defined. Even if space is represented only qualitatively rather than quantitatively, the set of movements in canonical directions still constitutes such a system. For example, a movement to the left reverses a movement to the right. However, the set of positive integers used here to represent distance does not constitute such a system within the representational system of space.

Carlson (1997) suggested that mental operations across domains have characteristic information-processing dynamics such that instantiating a goal to apply an operation evokes a procedural frame to which objects are assimilated. For example, forming an intention to add evokes a 
frame to which numerical operands are assimilated to perform addition. Sohn and Carlson (1998) found support for this hypothesis in the domains of arithmetic and Boolean logic. Carlson and Sohn (2000) extended this hypothesis to the imagined navigation task used in the present study. Their results supported a mapping of spatial directions to mathematical operations and distances to numerical operands. The major result in these studies was that over a range of conditions, individuals performed both symbolic and spatial tasks more fluently when operators (mathematical operations or spatial directions) were available in advance of operands (numbers or distances).

The spatial task used by Carlson and Sohn (2000) differed in an important way from real-life navigation, in that participants always interpreted directions with respect to their ecological frame of reference as they sat in front of the computer screen. Thus, although the starting location or origin for each step was a function of previous steps, the starting orientation was constant. In contrast, real-life navigation entails updating of one's frame of reference-both origin and orientation - with changes in heading that result from following directions. The present experiments extend the Carlson and Sohn paradigm to a situation in which individuals must update their orientations at each problem-solving step. We predicted that the benefit of considering direction in advance of distance would be found in both constant-frame (i.e., when the reference frame used to interpret spatial terms remains fixed throughout the task) and updated-frame (i.e., when the reference frame needs to be reoriented in each movement step) versions of the task.

However, an alternative account holds that the directionfirst benefit depends on the availability of a constant frame of reference for interpreting directions. On this account, in the constant-frame task, direction serves to select a particular set of contiguous locations that constitute a path from the current position (because orientation is constant and thus predetermined), and distance is used to select among this small set of possible locations. Distance alone, however, can only select a set of noncontiguous locations more difficult to hold in memory, thus resulting in less fluent performance than in the direction-first condition. In the updated-frame task, however, direction and distance might both serve as parameters of a single action of updating one's oriented location. If so, there is no reason to expect an advantage of considering one parameter in advance of another. This alternative is similar to the hypothesis that operators and operands in arithmetic serve similar roles as parameters of a retrieval process (e.g., Reder $\&$ Schunn, 1996). If this is the case, Carlson and Sohn's evidence for a common structure across domains would be limited to the constant-frame task, because only in that case would direction specify an action (following a path) parameterized by a distance.

\section{Experimental Task}

All studies that have provided evidence for the use of spatial frameworks have involved essentially the same task: locating objects in a mental model of a scene. The present experiment examined whether the spatial framework results are specific to this task or whether they can be obtained with other tasks that make use of the space around the body. If the latter is the case, this would provide additional evidence for the spatial framework model as an account of how people conceptualize the space that surrounds their body. Our tasks involve using the immediate space without forcing the participants to keep active in working memory any information other than that of their imagined location and, in some conditions, imagined heading. The experiments reported by Tversky and her colleagues (e.g., Franklin \& Tversky, 1990) required that participants remember direction-object associations and retrieve them during the test phase. It is not clear therefore whether the times needed to retrieve these associations are indicative of how the participants represented the spatial aspects of their mental models. De Vega, Rodrigo, and Zimmer (1996) used a similar task but modified the mode of response (i.e., they asked participants to use arrows to point to the objects) and showed that the spatial framework pattern of results was no longer obtained. This, along with evidence from Carr and Roskos-Ewoldsen (1999), suggests that the locus of the spatial framework results might be at the stage of retrieval.

In our experiments, we eliminated the need for people to remember and retrieve direction-object pairs. The only information they needed to hold was about their own imagined position in the space. Our paradigm thus involves a more direct online measure of how people use the space around them. The time participants take to perform the movement at each step is taken as a measure of the accessibility of the direction moved on that step. Thus, our measure reflects the time needed to perform an imagined spatial action in a simple and perceptually available environment, rather than in an elaborate environment that is held in memory.

We modified slightly the path construction task used by Carlson and Sohn (2000). This task involves step-by-step presentation of directions and distances that people use to construct a path through a checkerboard grid. Figure 1 presents the starting displays along with an example of the first step of a trial. Participants in this task imagine themselves moving to the four directions (front, back, left, and right) without locating or manipulating any objects. The task is similar to the one used by Vecchi and Girelli (1998), although we used a bigger grid that remained visible during the construction of the path. In the original task (Carlson \& Sohn, 2000), participants received direction and distance information at each of four steps. The direction was indicated by one of the letters A, B, L, R, corresponding to above, below, left, and right. Directions were always given with respect to the participants' ecological reference frame as they were seated in front of a computer, and this frame remained constant throughout the experiment. Interpreting directions with a constant reference frame makes use of the space around the participant's body (ecological reference frame) but not the space around the participant's 
imagined position on the grid (egocentric frame). In Experiment 1 , in addition to a modified version of the constantframe condition ${ }^{1}$ (henceforth constant-orientation), we included an updated-orientation condition, in which we instructed participants to interpret each direction on the basis of their current imagined heading (Figure 2). To perform the task, participants thus had to update the orientation of their reference frame on most of the steps of the problem (i.e., in all steps except when front was presented).

\section{EXPERIMENT 1}

In one condition of Experiment 1, participants were asked to perform the path construction task by updating, when needed, their imagined orientation. If the spatial framework model describes the interpretation of directions in this task, then the time required to move in each direction will follow the spatial framework pattern of results: Imagined movements to the front will be on average faster than those to the back, which in turn will be faster than movements to either the left or right. If instead imagined movements are described best by a mental rotation account (Kosslyn, 1994), then the movements to the back should take longer than movements to the left or right because of the greater angle of mental rotation. As a control, we included a condition similar to the original path construction task that Carlson and Sohn (2000) used in their study. In this condition, directions were given on the basis of a constant frame of reference; therefore, participants did not need to maintain an updated orientation to interpret them. Because spatial frameworks studies (e.g., Franklin \& Tversky, 1990) have involved occasional updating of orientations, we were interested in assessing whether the pattern of movement times for this condition would also exhibit the spatial framework pattern.

\section{Method}

Participants. Forty-six students from psychology classes at the Pennsylvania State University participated in this experiment in exchange for course credit. Twenty-three participants ( 9 female and 14

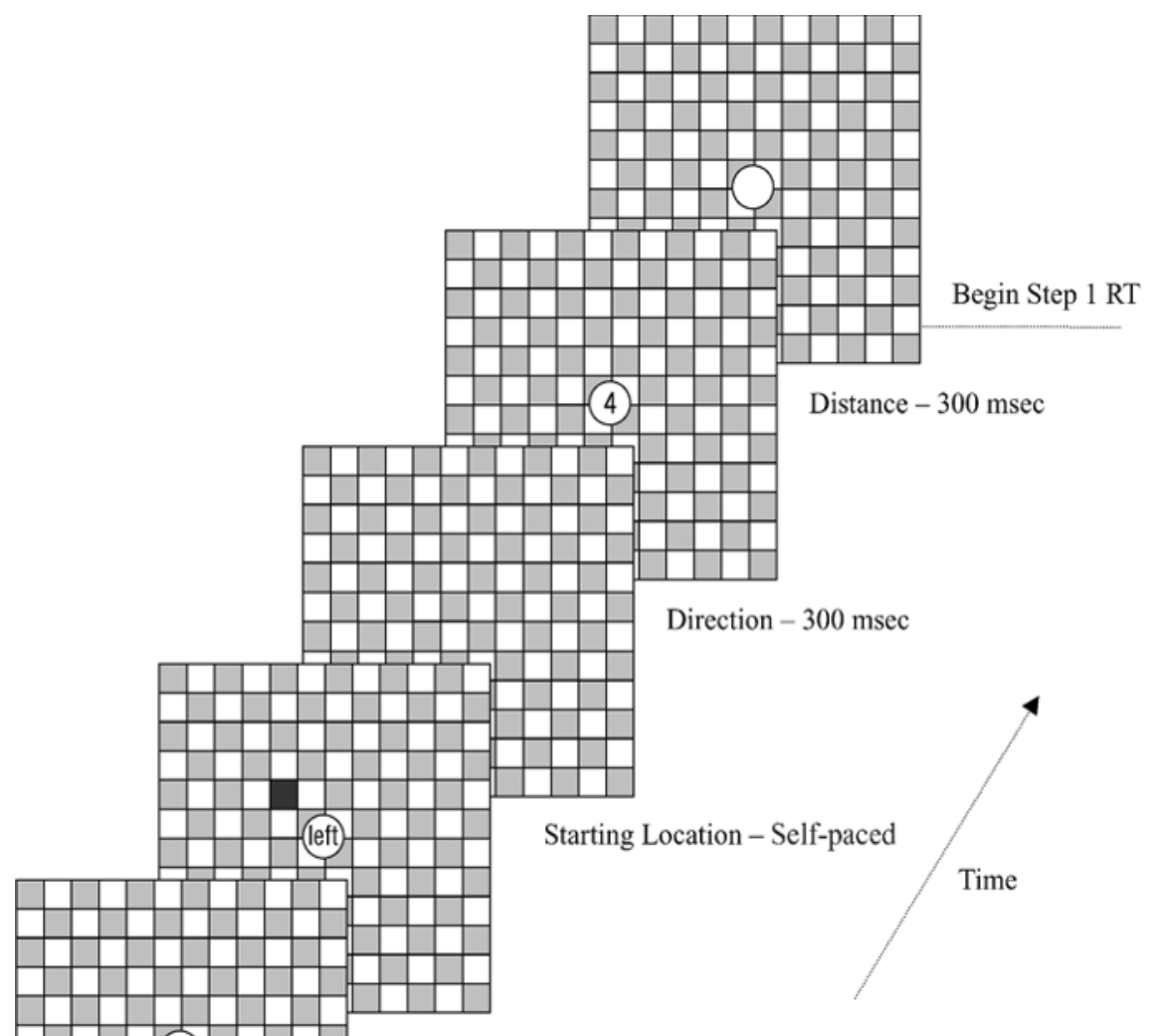

Begin Trial - Self-paced

Figure 1. The course of the first step of a direction-first trial of the experimental task. The starting location display that contains the black square (the square was red in the actual experiments) was presented only before the first step. Distance-first trials were identical with the exception that the distance display preceded the direction display. In this example, the participants should imagine moving four squares to the left of the starting square. 

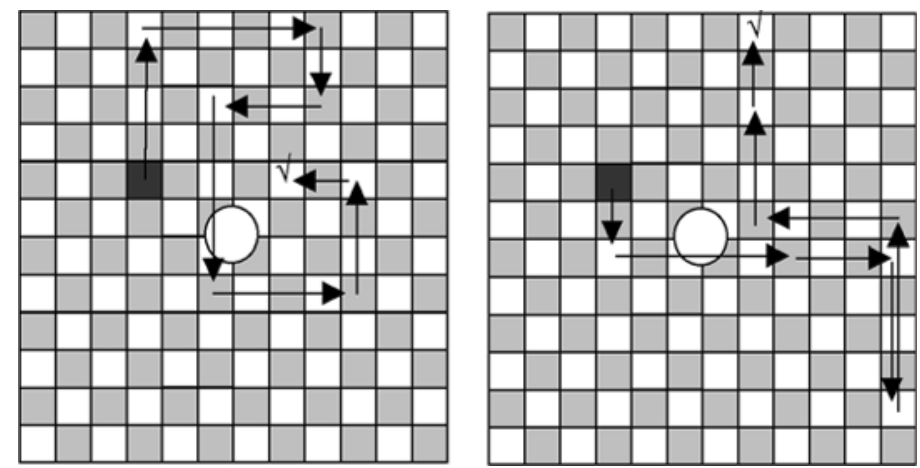

Figure 2. Navigation examples for the two orientation conditions of Experiment 1 . The constant-orientation example (left side) reflects the following sequence of directions: front 4 , right 5 , back 2 , left 3 , back 5 , right 4 , front 3 , left 2 . The updated-orientation example (right side) presents the following sequence: back 2 , left 5 , front 3 , right 4 , back 5 , left 4 , right 3 , front 2 . Starting and ending locations are shown with a dark square and a check-mark, respectively.

male) were randomly assigned to each of the orientation conditions. Assignment of participants to conditions was random but it was performed independently for males and females to ensure equal proportions of each gender in each condition. All but 2 of the participants were right-handed. The 2 left-handed participants reported that they were capable of using a computer mouse with their right hand.

Materials. The stimuli were presented on 19-in. Dell color monitors controlled by Dell Optiplex microcomputers equipped with Intel Pentium III processors. The monitors were placed lying on their backs on custom-made stands. Participants were seated at a comfortable distance of about $50 \mathrm{~cm}$ from the center of the screen and viewed the display from an angle that ranged between $45^{\circ}$ and $60^{\circ}$ depending on the height of the participant. A computer mouse was placed on a table to the right of the monitor, at the same height as the display.

Each display included the presentation of a $12 \times 12$ grid that alternated between light- and dark-filled squares. Each square's side was approximately $1.3 \mathrm{~cm}$. A green circle with a diameter of $1.3 \mathrm{~cm}$ was displayed at the center of the grid. All of the information about direction and distance was presented in the center of the circle.

Design. Experiment 1 featured a 2 (task: constant-orientation, updated-orientation) $\times 2$ (order of information: direction-f irst, distance-first) $\times 4$ (direction: front, back, left, right) mixed factorial design. Task was manipulated between participants; both order of information and direction were manipulated within participants.

Procedure. Each participant completed two practice trials and then five blocks of 12 problems. Each problem required participants to mentally move around the grid, using four directions (front, back, left, and right), and distances of two to five squares. Each direction was randomly sampled twice in each eight-step problem. The value of distance was randomly selected at each step. Participants were instructed to trace a path within the grid by imagining themselves on it. Specifically, they were asked to think of the grid as a tiled floor and imagine themselves walking on it. Those in the updated-orientation condition were instructed to interpret each direction on the basis of their imagined heading, whereas those in the constant-orientation condition were instructed to do so on the basis of their own reference frame (i.e., "left" meant their own left regardless of their imagined orientation in the grid).

Each problem began with the presentation of the grid. Initially, a pound sign was displayed in the center of the green circle. Participants clicked with the mouse (whose cursor was not visible) to request the starting location, which appeared as a red square. Starting locations were chosen randomly for each problem, with the con- straint that they never appeared in the outermost cells of the grid. Participants clicked again to request the direction-distance information. The order in which directions and distances appeared varied randomly from problem to problem but it remained constant within each problem. In half of the trials, the directions preceded the distances; in the other half, the opposite was the case. In contrast to Carlson and Sohn (2000), whole words (i.e., front, back, left, and right) were used to indicate directions. On the basis of previous experiments (Carlson \& Sohn, 2000), directions and distances were set to appear for $300 \mathrm{msec}$ each.

Within each problem, eight steps of movement were performed. Intermediate locations were not marked on the screen; they were instead maintained mentally. Participants were instructed not to use their hands to mark the locations. At the end of the eighth step, a checkmark appeared at the center of the green circle, and participants used the mouse to move the check-mark and click on the ending location of the path to enter their response. Participants were instructed to perform the task as fast as they could without sacrificing accuracy for speed.

\section{Results}

The first block of trials was considered practice and was not included in any of the analyses. Three participants from the updating-orientation condition were excluded from all analyses because of low accuracy (less than $20 \%$ correct). Participants answered correctly approximately $74 \%$ of the problems. Accuracy did not differ for the constant $(75 \%)$ and the updating $(73 \%)$ orientation conditions $\left[F(1,41)=0.72, M S_{\mathrm{e}}=0.0564 ; \alpha=.05\right.$ for all reported analyses]. Similarly, there was no difference in accuracy between the direction-first (74\%) and distance-first conditions $(74 \%)\left[F(1,41)=0.03, M S_{\mathrm{e}}=0.0069\right]$, and the task $X$ order of information interaction was not significant $\left[F(1,41)=2.06, M S_{\mathrm{e}}=0.0069\right]$. The rest of the analyses reported here are based on the latency data for problems with correct responses.

All step times were recorded from the time that all of the information to complete the step became available. Timing thus began when the distance appeared in the direction-first trials and when the direction appeared in the distance-first trials and ended when participants clicked to request a new direction-distance pair or, in the case of 


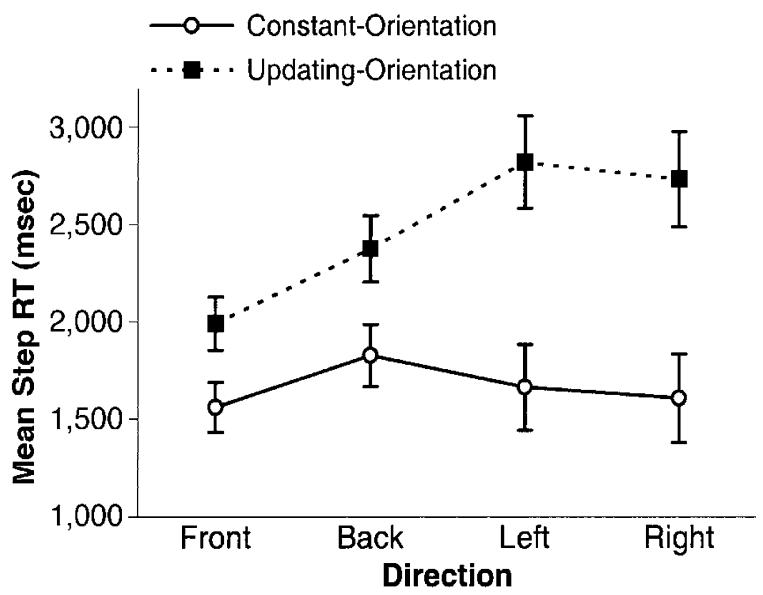

Figure 3. Mean step latency in Experiment 1 as a function of task and direction. Error bars indicate standard errors.

the eighth step, to get the check-mark. Latency analyses revealed that the average step time was shorter in the constant-orientation condition $(1,665 \mathrm{msec})$ than in the updated-orientation condition $(2,480, \mathrm{msec})[F(1,41)=$ 9.92, $\left.M S_{\mathrm{e}}=5,726,107, p<.01\right]$.

As predicted by the procedural frame hypothesis, there was a significant main effect for the order of information $\left[F(1,41)=6.25, M S_{\mathrm{e}}=57,998, p<.05\right]$. Participants took on average less time to perform a step when the direction was presented first $(2,040 \mathrm{msec})$ than when the distance was first $(2,105 \mathrm{msec})$. This operator-first benefit was similar in magnitude in both the updated-orientation and the constant-orientationtask, as implied by the nonsignificant task $\times$ order of information interaction $[F(1,41)=$ $\left.0.22, M S_{\mathrm{e}}=57,998\right]$.

A significant task $\times$ direction interaction was evident in the data $\left[F(3,123)=11.56, M S_{\mathrm{e}}=266,241, p<.001\right]$. A simple main effect of direction was significant in both tasks [constant-orientation, $F(3,66)=6.60, M S_{\mathrm{e}}=94,036$, $p<.001$; updated-orientation, $F(3,57)=12.28, M S_{\mathrm{e}}=$ $465,637, p<.001]$. Average step times for each direction are presented in Figure 3 as a function of task condition.

As expected, in the updated-orientation task, times for each direction replicated the spatial framework pattern. Like all other $t$ tests in this article, these were pairwise two-tailed Bonferronitests. The times for front $(1,992 \mathrm{msec})$ were significantly faster than the times for back $(2,375 \mathrm{msec})[t(19)=-4.25, p<.001]$, which in turn were faster than the times for left $(2,820 \mathrm{msec})$ and right $(2,732 \mathrm{msec})[t(19)=-3.08, p<.01$, and $t(19)=-2.34$, $p<.05$, respectively]. The times for left and right did not differ significantly from each other $[t(19)=-0.85]$.

However, the average step times in the constantorientation task did not follow the spatial-framework pattern. Instead, the time to move to a particular direction depended on the angular deviation of the direction from front. The mean step time for front $(1,561 \mathrm{msec})$ was again the shortest, but the mean time for back $(1,828 \mathrm{msec})$ was longer than the times for left $(1,664 \mathrm{msec})$ and right
$(1,608 \mathrm{msec})[t(22)=2.13, p<.05$, and $t(22)=3.03$, $p<.01$, respectively]. This pattern of movement times can be best accounted for by mental rotation.

To provide a check on random assignment, we analyzed the times to initiate problems. Initiation time $(1,357 \mathrm{msec})$ was not significantly different between groups $[F(1,41)=$ $\left.0.001, M S_{\mathrm{e}}=4,597,553\right]$. The task $\times$ order of information interaction was also nonsignificant $[F(1,41)=0.62$, $\left.M S_{\mathrm{e}}=359,092\right]$, and no main effect for the order of information was observed $\left[F(1,38)=0.93, M S_{\mathrm{e}}=359,092\right]$.

Finally, the time participants took to enter their responses at the end of each eight-step trial $(984 \mathrm{msec})$ did not differ between the two tasks $\left[F(1,41)=0.012, M S_{\mathrm{e}}=\right.$ $50,616]$. Similarly, nothing else was significant for this measurement (all $F$ s $<0.6)$.

\section{Discussion}

Experiment 1 revealed that the spatial framework pattern of results is not limited to tasks that involve locating objects in an imagined environment. The pattern of times in the updated-orientation condition replicated the spatial framework pattern, showing that the spatial frameworks can apply for tasks that involve other spatially guided actions, such as movements to the various directions in an imagined environment. This result suggests that the spatial framework pattern is not produced by processes involved in the retrieval of object-location associations.

Results from the constant-orientationtask deviated significantly from the standard pattern predicted by spatial frameworks. Instead, the times conformed to the prediction of a mental rotation account.

Perhaps the increased latencies with movements to the left and right in the updated-orientation condition were due to the fact that, in that version of the task, participants constantly experienced difficulties in telling left from right. The mappings of spatial labels to regions of space changed depending on the participants' imagined orientation, and therefore participants had to perform spatialterm assignment at almost every step of a problem in that condition. Moreover, no cues in the environment could have been used reliably to make spatial-term assignment easier. In view of Maki et al.'s (1979) finding that the people's difficulty with left and right is caused by difficulties in spatial-term assignment, we believe that in the updatedorientation conditions participants experienced this difficulty on every step.

In the constant-orientation condition, the mapping of spatial labels to regions of space was fixed throughout the experiment. Therefore, participants needed to map the labels at the appropriate regions of space only once and then simply retrieve (by possibly using cues in the environment) the assignment of spatial terms to regions of space. It is possible, however, that the difficulty with left and right was reduced gradually in the constant-orientation condition. If this was the case, one would expect to find the spatial framework pattern of latencies during the first blocks of the constant-orientation condition. However, separate statistical analyses for each block of the constant- 
orientation revealed that this was not the case. The pattern predicted by the mental rotation account was obtained in all four experimental blocks of the constant-orientation condition, and was even stronger in the practice block $\left[F(3,66)=4.20, M S_{\mathrm{e}}=221,771, p<.01\right]$.

In all previous studies supporting the spatial framework model (e.g., Bryant \& Tversky, 1992; Bryant et al., 1992; Bryant \& Wright, 1999; Franklin \& Tverksy, 1990), the participant was occasionally reoriented in the mental model. Similarly, in our experiment, the spatial framework pattern was obtained only when participants occasionally updated their orientation. It may be, then, that occasional updating is a condition for the spatial framework model.

The results of Experiment 1 also provided additional support for the procedural frame hypothesis. One might predict that in the case of our updated-orientationtask, in which a direction has to be maintained as a heading in working memory, people would have more difficulty with the direction-first trials because they would have to maintain another direction in working memory until the distance became available. However, our results revealed an operator-first benefit in both the constant- and updatedorientation conditions.

An alternative explanation for the different effects of direction on step time in the constant- and updatedorientation conditions is that participants in the constantorientation condition performed the task using a reference frame supported by the environment which was thus less demanding of working memory resources. It is possible that with more working memory capacity at their disposal, participants in the constant-orientation condition could have made the difficult left versus right discrimination more easily. The goal of Experiment 2 was to test this alternative hypothesis by reducing the cognitive demands of the updating-orientation condition.

\section{EXPERIMENT 2}

In Experiment 2 we manipulated the external support for frame of reference information and thus the working memory demands of updating one's reference frame. The goal of this experiment was to examine whether the difference in the pattern of step latencies between the constant- and updated-orientation conditions in Experiment 1 was driven by the lack of external support and hence the increased working memory demands in the updated-orientation condition.

In Experiment 1, participants in the updated-orientation condition had to update an egocentric reference frame at each step of a problem. For most of the steps in the experiment, they had to imagine themselves at orientations inconsistent with the actual orientations of their ecological reference frame (without having any external support for their imagined orientation). In contrast, participants in the constant-orientation condition have used a reference frame that remained aligned with their own ecological reference frame. Presumably, this difference in external support produced different demands on working memory for the two conditions. To perform the task correctly, participants in the updated-orientation condition need to maintain information about their current heading. This demand is absent from the constant-orientation condition because the heading is constant throughout the experiment. Perhaps, then, in this condition, the available working memory resources enabled participants to carry out efficiently the task of mapping the terms left and right to the appropriate regions of space. This could explain why movement times for left and right were relatively fast in the constantorientation condition.

In Experiment 2, we repeated the updated-orientation condition with two variations. In one version, we required that participants mark their intermediate locations. This condition reduces working memory load by eliminating the need to maintain intermediate locations in working memory but does not provide any external support for the orientation of the egocentric frame. In the second variation, participants marked both the intermediate locations and the current orientations of the egocentric frame. This condition provides external support for the orientation of the egocentric frame and offers an additional reduction in working memory load by eliminating the need to hold heading information in working memory. The availability of additional working memory resources could enable participants to discriminate left and right more easily. Therefore, if the constant-updating latency of Experiment 1 was due to low working memory demands, then the mental rotation pattern (i.e., left and right faster than back) should be observed in Experiment 2. However, if the pattern was caused by the fact that a constant reference frame was used to interpret the spatial directions, then both conditions of Experiment 2 should produce the updatedorientation pattern of step latencies.

\section{Method}

Participants. Participants were 42 psychology students from Pennsylvania State University. None had participated in Experiment 1. Half of the participants, 11 male and 10 female, were assigned to each memory condition. Five of our participants were left-handed but they all reported being capable of using a computer mouse with their right hand.

Materials. The physical setup of Experiment 2 was similar to that of Experiment 1. In fact, the only difference was that in Experiment 2 a computer keyboard was placed on the lap of participants and was used to mark intermediate locations. Drawings of arrows pointing to each of the four canonical horizontal directions (i.e., front, back, left, and right) covered four keys of the numeric keypad on the keyboard ("8," " 2 ," " 4 ," and "6," respectively, chosen because they form a cross). The arrows were black on yellow backgrounds that covered the whole surface of the keys. All other keys of the keyboard were disabled.

Design. Experiment 2 featured a 2 (memory aid: location aid, location plus orientation aid) $\times 2$ (order of information: directionfirst, distance-first) $\times 4$ (direction: front, back, left, right) mixed factorial design. Memory aid was manipulated between participants whereas both the order of information and direction were manipulated within participants.

Procedure. In both memory aid conditions, participants clicked the mouse to begin a trial. In contrast to Experiment 1, after the direction-distance pair appeared, a mouse cursor became visible in the center of the screen. Participants were instructed to imagine 


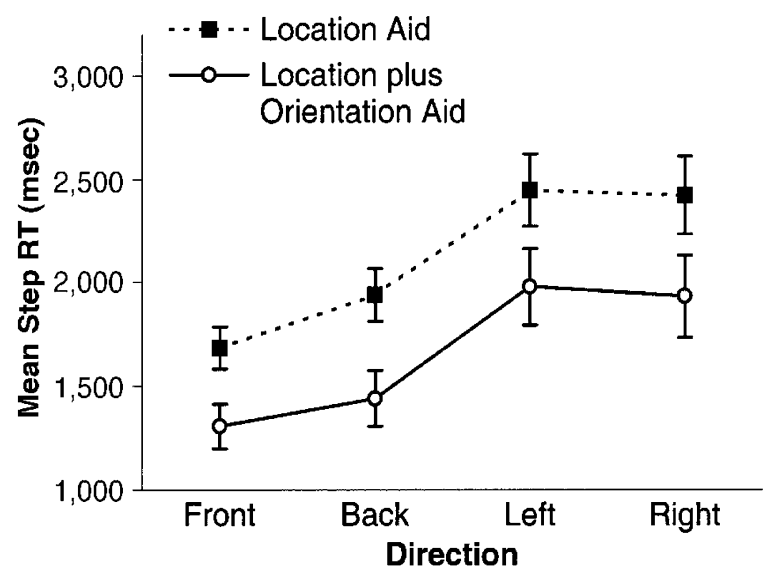

Figure 4. Mean step latency in Experiment 2 as a function of task and direction. Error bars indicate standard errors.

themselves moving on the grid on the basis of the direction and distance they received, and then to click with the mouse on the square at which they ended. When they did so, the computer beeped once and the cursor disappeared. Timing began when the second piece of information in the direction-distance pair appeared and stopped as soon as participants clicked on the intermediate location. This was the main dependent measure and will henceforth be referred to as step latency. After the beep, participants pressed the arrow key that corresponded to their imagined facing direction at the end of the step. In the location plus orientation aid condition, the square that participants had previously clicked on became red and a yellow arrow pointing to the direction they had selected appeared on the square. In the location aid condition, the square became red but no arrow appeared on it. The time between clicking on the intermediate location and pressing the arrow key was recorded and will be referred to as marking latency. After marking their location or location plus orientation, participants clicked to request a new directiondistance pair. The time between pressing the arrow key and requesting the information for the subsequent test was also recorded and will be referred to as request latency.

At the end of the eighth step, a check-mark appeared on the last marked location and participants were simply asked to click on it to enter their response.

As in Experiment 1, participants were asked to perform the task as quickly as possible without sacrificing accuracy for speed. Moreover, participants received the same imagery instructions as in the updated-orientation condition of Experiment 1.

\section{Results}

Data from 3 participants, 2 from the location aid condition, were discarded because of low accuracy (less than $20 \%$ correct). As in Experiment 1, the first block of trials was considered practice and was excluded from all analyses.

Participants answered correctly approximately $85 \%$ of the problems, thus performing the task more accurately than the participants of Experiment 1. This proportion did not differ significantly between the location aid $(84 \%)$ and the location plus orientation $(87 \%)$ conditions $[F(1,36)=$ $\left.0.61, M S_{\mathrm{e}}=0.0256\right]$. Similarly, no accuracy difference was obtained between the operator-first and the operandfirst conditions $\left[F(1,36)=0.33, M S_{\mathrm{e}}=0.0052\right]$. No significant memory aid $\times$ order of information interaction was obtained $\left[F(1,36)=0.68, M S_{\mathrm{e}}=0.0052\right]$. Remaining analyses will focus on latency data.

The mean step latency for the location plus orientation memory aid $(1,664 \mathrm{msec})$ was significantly shorter than that for the location aid condition $(2,123 \mathrm{msec})[F(1,36)=$ $\left.4.92, M S_{\mathrm{e}}=3,244,866, p<.05\right]$. Furthermore, step latencies for the location plus orientation condition were almost identical to those recorded in Experiment 1 for the constant-orientation condition and considerably shorter than those for the updated-orientation condition.

Replicating the results of Experiment 1, the present experiment revealed a significant main effect for the order of information $\left[F(1,36)=36.61, M S_{\mathrm{e}}=24,259, p<.001\right]$. Trials with the operator presented first $(1,840 \mathrm{msec})$ elicited shorter step latencies than trials with the operand presented first $(1,947 \mathrm{msec})$. The order of information did not interact with either memory aid $[F(1,36)=0.02$, $\left.M S_{\mathrm{e}}=24,259\right]$ or direction instruction $[F(3,108)=1.48$, $\left.M S_{\mathrm{e}}=31,115\right]$. Furthermore, the memory aid $\times$ direction interaction was not significant $\left[F(3,108)=0.39, M S_{\mathrm{e}}=\right.$ 152,777].

However, a main effect for direction was obtained $\left[F(3,108)=63.29, M S_{\mathrm{e}}=152,777, p<.001\right]$ (Figure 4). For both memory aid conditions, the spatial framework pattern of results was obtained. Step latencies for front $(1,495 \mathrm{msec})$ were shorter than those for back $(1,690 \mathrm{msec})$ $[t(37)=-6.09, p<.001]$, which in turn were shorter than the times for left $(2,213 \mathrm{msec})$ and right $(2,177 \mathrm{msec})$ $[t(37)=-8.12, p<.001$, and $t(37)=-6.95, p<.001$, respectively]. No significant difference was obtained between the times for left and right $[t(37)=-0.96]$.

The analysis of marking latencies $(450 \mathrm{msec})$ revealed that after participants performed a movement to the front, they were faster pressing the corresponding arrow key (357 $\mathrm{msec})$ than after moving to any other direction (481, 487 , and $478 \mathrm{msec}$, respectively, for back, left, and right) $\left[F(3,108)=15.90, M S_{\mathrm{e}}=18,730, p<.001\right]$. Indeed, pairwise $t$ tests showed that the differences between front and the other directions were statistically reliable (all $p$ s $<$ .001). Presumably, this occurred because after moving to the front participants simply needed to repeat the response of the previous step. Moreover, participants were faster pressing an arrow key in the location plus orientation task (405 msec) than in the location task (496 msec), but this difference was not significant $\left[F(1,36)=1.62, M S_{\mathrm{e}}=\right.$ $390,925]$. No other effects on marking latency were close to being significant (all $F_{\mathrm{S}}<1$ ). Similarly, no significant effects were found for request latency (454 msec; all $F \mathrm{~s}<1)$.

In addition, time to initiate problems did not vary across the memory aid groups $\left[F(1,36)=0.88, M S_{\mathrm{e}}=373,739\right]$ or the order of information conditions $[F(1,36)=1.36$, $\left.M S_{\mathrm{e}}=24,956\right]$, thus providing a check on random assignment.

Finally, there was no effect of memory aid on the time to enter the response after the end of the eighth step $(453 \mathrm{msec})\left[F(1,36)=0.04, M S_{\mathrm{e}}=56,670\right]$. The time to enter the response in Experiment 2 was substantially shorter 
than the corresponding time recorded in Experiment 1. This was probably due to the fact that in Experiment 2 the cursor appeared on the last marked location instead of the center of the screen, eliminating movement time.

\section{Discussion}

The results of Experiment 2 replicated the pattern of latencies obtained in the updated-orientation condition of Experiment 1. The spatial framework pattern of results was evidenced in both conditions of the present experiment. Even when we provided external support for the reference frame that is used to perform the task and made the task less demanding in working memory resources, the results still followed the spatial framework pattern. This suggests that it was neither the external support nor the lower working memory demands of the constant-orientation condition that caused the differences between the two conditions in Experiment 1.

Replicating the results of Experiment 1 and previous studies (Carlson \& Sohn, 2000; Sohn \& Carlson, 1998), Experiment 2 provided support for the procedural frame hypothesis. Mean step times were significantly shorter when the directions appeared before the distances.

\section{GENERAL DISCUSSION}

If indeed the way we conceptualize our immediate space in imagined settings depends on the invariant characteristics of our bodies and the environment, then the spatial framework pattern of results should be obtained in any task that requires reasoning about immediate space. Previous studies (e.g., Bryant et al., 1992; Franklin \& Tversky, 1990) have documented the spatial frameworks pattern of results in experiments in which participants were asked to locate objects in imagined or remembered spaces. Our experiments extend the range of tasks to which the spatial framework model applies by providing empirical evidence that, in some cases, spatial framework results are also obtained when participants interpret spatial directions to perform imagined navigation. Unlike the tasks in previous studies, our tasks do not require storage in working memory and later retrieval of the locations of objects. Instead, they provide a more direct measure of how people make use of the space that surrounds their imagined self.

The pattern of step times diagnostic of spatial frameworks appeared only when participants had to update their imagined orientation at each step, suggesting that reorienting may be a condition for the application of the spatial framework model. In Experiment 1, the constantorientation condition, which required only a translation (and not a rotation) of the reference frame, produced results that varied from the ones that the spatial framework model predicts. Experiment 2 showed that the spatial framework pattern of results is due neither to the absence of external support for the frame nor to increased working memory demands. Providing external support for the frame, which also reduced working memory demands, did not significantly change the pattern of results, suggesting that the updating process is responsible for the observed results.

How exactly the updating process produces these results remains to be explored. One possibility is that because of the updating processes, participants needed to repeat the assignment of spatial labels to the appropriate regions of space at each step. This constant reassignment of spatial labels may have prevented participants from using cues of the environment to break the symmetry of the left/right axis. As a result, participants had substantial difficulty discriminating left from right throughout the experiment. Support for the plausibility of this hypothesis is provided by past research. Specifically, a number of studies (e.g,. Artez, 1991; Hintzman, O'Dell, \& Arndt, 1981; Shepard \& Hurwitz, 1984) have documented that people are slower to decide between left and right when their egocentric reference frame is not aligned with the environmental frame in which the decision must be made (e.g., when deciding whether to turn left or right on the basis of a nonrotatable north-up map while traveling south). Similarly, Avraamides (2002), in a study that uses the spatial framework paradigm, provides evidence that participants have greater difficulty discriminating left from right in the conditions in which their ecological and egocentric frames are misaligned. It might be the case, then, that spatial frameworks reflect the characteristics of the continuous reassignment of verbal labels to particular regions of space.

Indeed, empirical evidence suggests that the accessibility of spatial directions is different when participants are asked to point to objects instead of labeling the directions they occupy (De Vega et al., 1996). De Vega and Rodrigo (2001) showed that the internal spatial framework pattern is not obtained when people point to the locations of objects after having performed physical rotations. They proposed that although the use of spatial terms is anchored in a mental framework from which the ecological self is detached, pointing is bound to a sensorimotor framework (i.e., a mental representation that is organized on the basis of the ecological self). It is possible, then, that our constantorientation condition elicited the use of a sensorimotor framework (because the directions were interpreted on the basis of the participants' ecological reference frame), producing results that deviated from internal spatial frameworks. In contrast, the use of deictic terms in the updatingorientation condition could have invited the use of a mental framework that gave rise to internal spatial frameworks.

An alternative explanation for the results of Experiment 1 is that, despite the experimental instructions, participants in the constant-orientation condition did not adopt perspectives that were embedded in the grid. Although informal postexperiment interviews with our participants suggested that this was not the case, we acknowledge this possibility and we encourage future work to address it.

In addition to generalizing the spatial frameworks model to imagined navigation, our experiments provide 
evidence for the mapping of directions and distances to operators and operands, respectively. The distinction between operators and operands, which has been previously documented in the domain of mental arithmetic (Carlson \& Sohn, 2000; Sohn \& Carlson, 1998), has been shown here to hold for a spatial path construction task that resembles navigation of real environments. Our results have provided additional evidence for the procedural frame hypothesis that posits a special role of operators in the execution of mental activity. In all conditions of our two experiments, the time needed to complete a step of the task was shorter when the operators preceded the operands, suggesting that operators serve the function of instantiating goals that provide a reference frame for assimilating the operands. The advantage documented when the goalrelated information precedes the operands suggests that there is a preferred order in which information is processed. The operator-first benefit was present with both our constant-orientation condition-replicating the results of Carlson and Sohn-and our updated-orientation condition, which is more similar to real-life navigation. Furthermore, the benefit was not affected by the presence of external support for the reference frame and the lower demands on working memory resources. Our results show that the application of the procedural frame hypothesis is not limited to the mental arithmetic domain or to spatial tasks that do not require reorientation.

The present results indicate that imagined navigationwhich requires updating of orientation-involves mental processes that are organized egocentrically, in that the imagined self serves as an origin for a frame of reference organized in terms of imagined bodily axes. These processes also appear to be organized in terms of the operatoroperand structure postulated by the procedural frame hypothesis (Carlson, 1997; Sohn \& Carlson, 1998). If these results generalize to richer depicted spaces, such as real maps, they may also have practical applications in reallife tasks that require individuals to integrate verbal directions and depicted spaces to generate spatial activity.

\section{REFERENCES}

Artez, A. J. (1991). The design of electronic map displays. Human Factors, 33, 85-101.

AvraAmides, M. N. (2002). Spatial updating of environments described in texts. Manuscript submitted for publication.

Bryant, D. J., LANCA, M., \& Tversky, B. (1995). Spatial concepts and perception of physical and diagrammed scenes. Perceptual \& Motor Skills, 81, 531-546.

Bryant, D. J., \& Tversky, B. (1992). Assessing spatial frameworks with object and direction probes. Bulletin of the Psychonomic Society, 30, 29-32.

Bryant, D. J., Tversky, B., \& Franklin, N. (1992). Internal and external spatial frameworks for representing described scenes. Journal of Memory \& Language, 31, 74-98.

Bryant, D. J., \& WRIGHT, G. W. (1999). How body asymmetries determine accessibility in spatial frameworks. Quarterly Journal of Experimental Psychology, 52A, 487-508.
CARLson, R. A. (1997). Experienced cognition. Mahwah, NJ: Erlbaum. CARlson, R. A., \& Sohn, M.-H. (2000). Cognitive control of multistep routines: Information processing and conscious intentions. In S. Monsell \& J. Driver (Eds.), Control of cognitive processes: Attention and performance XVIII (pp. 443-464). Cambridge, MA: MIT Press.

CARR, W., \& Roskos-EWoldSEn, B. (1999). Spatial orientation by mental transformation. Psychological Research, 62, 36-47.

Chapman, M. (1988). Constructive evolution: Origins and development of Piaget's thought. Cambridge: Cambridge University Press.

Clark, H. H. (1973). Space, time, semantics, and the child. In T. Moore (Ed.), Cognitive development and the acquisition of language ( $\mathrm{pp} .27-$ 63). New York: Academic Press.

Corballis, M. C., \& Beale, I. L. (1976). The psychology of left and right. Hillsdale, NJ: Erlbaum.

De Vega, M., \& Rodrigo, M. J. (2001). Updating spatial layouts mediated by pointing and labelling under physical and imaginary rotation. European Journal of Cognitive Psychology, 13, 369-393.

De Vega, M., Rodrigo, M. J., \& Zimmer, H. (1996). Pointing and labeling directions in egocentric frameworks. Journal of Memory \& Language, 35, 821-839.

Franklin, N., \& Tversky, B. (1990). Searching imagined environments. Journal of Experimental Psychology: General, 119, 63-76.

Hintzman, D., O'Dell, C., \& ARndt, D. (1981). Orientation in cognitive maps. Cognitive Psychology, 13, 149-206.

Jeannerod, M. (1995). Mental imagery in the motor context. Neuropsychologia, 33, 1419-1432.

KossLyn, S. M. (1987). Seeing and imagining in the cerebral hemispheres: A computational approach. Psychological Review, 94, 148175.

KossLyn, S. M. (1994). Image and brain: The resolution of the imagery debate. Cambridge, MA: MIT Press.

MAKI, R. H. (1979). Processing relative locations in a natural space. Bulletin of the Psychonomic Society, 14, 25-28.

MaKi, R. H., Grandy, C. A., \& Hauge, G. (1979). Why is telling right from left more difficult than telling above from below? Journal of Experimental Psychology: Human Perception \& Performance, 5, 52-67.

Piaget, J. (1954). The construction of reality in the child. New York: Ballantine.

Reder, L. M., \& Schunn, C. (1996). Metacognition does not imply awareness: Strategy choice is governed by implicit learning and memory. In L. M. Reder (Ed.), Implicit memory and metacognition (pp. 4577). Mahwah, NJ: Erlbaum.

Shepard, R., \& Hurwitz, S. (1984). Upward direction, mental rotation, and discrimination of left and right turns on maps. Cognition, 18, 161-193.

Sohn, M.-H., \& CARLSON, R. A. (1998). Procedural frameworks for simple arithmetic skills. Journal of Experimental Psychology: Learning, Memory, \& Cognition, 24, 1052-1067.

Tversky, B., Morrison, J. B., Franklin, N., \& Bryant, D. J. (1999). Three spaces of spatial cognition. Professional Geographer, 51, 516524.

VeCCHI, T., \& GirelLI, L. (1998). Gender differences in visuo-spatial processing: The importance of distinguishing between passive storage and active manipulation. Acta Psychologica, 99, 1-16.

\section{NOTE}

1. The differences from Carlson and Sohn's task were that we replaced above and below with front and back, communicated directions using the actual terms instead of the first letters of the terms (i.e., left instead of $L$ ), and presented the grid on a monitor rotated in depth to enable the use of front and back (i.e., with an upright monitor, front and back would have led to movements up and down the screen).

(Manuscript received September 12, 2001; revision accepted for publication October 15, 2002.) 